\title{
Free expansion of elastic filaments
}

\author{
A. J. Spakowitz and Z.-G. Wang \\ Division of Chemistry and Chemical Engineering, California Institute of Technology, Pasadena, California 91125
}

(Received 16 May 2001; revised manuscript received 13 July 2001; published 27 November 2001)

\begin{abstract}
The dynamics of an elastic polymer filament undergoing contour length expansion is studied using computer simulation. The expansion occurs by development of transverse buckling waves that grow through a coarsening process. The growing buckles locally organize into a helical structure with a characteristic persistence length. The helical domain boundaries are eliminated from the relaxing structure by unwinding through the ends of the rod. The growth of the helical domains results in self-propulsive motion of the expanding rod, as one large helix spanning the entire chain relaxes during the late stages of the dynamics. Stability analyses and scaling arguments are provided to explain the simulation results.
\end{abstract}

DOI: 10.1103/PhysRevE.64.061802

PACS number(s): 36.20.-r, 05.70.Ln, 31.70.Hq, 87.10.+e

\section{INTRODUCTION}

Many important biological and polymeric systems quickly respond to changes in their surrounding conditions by altering their conformations. These conformational adjustments include volume expansion in response to changes in the conditions of the solution ( $\mathrm{pH}$, temperature, salinity) and volume compression due to externally applied stress or intramolecular attraction. For example, anionic hydrogel [1] microspheres respond to changes in $\mathrm{pH}$ and salt concentration by expanding to a swelling ratio of as much as 12 . Microgels, which respond to these changes much faster than slab gels ( 0.5 seconds versus hours), are being evaluated as likely candidates for drug delivery systems because of their small size $(\sim 10 \mu \mathrm{m})$ and short response time. Similar gel chemistry is employed in the production of nanoscale conduits [2]. Single nanotubes can be formed from biomembrane vesicles by bonding a substrate to the membrane surface and drawing a stabilized fluid-lipid bilayer. The caliber of the nanometer scale tube can be experimentally controlled in the range 20 200 nanometers. The nanotube is stabilized by photochemical polymerization of cross-linking monomers contained within the lipid bilayer, resulting in a cross-linked gel cylinder of nanometer scale radius and near-millimeter length. When anionic hydrogel chemistry is employed, nanotubes with the capability of expanding their equilibrium lengths are produced. Nanoscale devices may use the expansion of these nanotubes for hydrodynamic propulsion or as mechanical levers, where adjustments in the solution conditions trigger the desired response. Understanding the dynamics of this expansion process is important for the design of nanoscale devices. Toward this goal, we simulate and analyze the free expansion of an initially straight elastic rod after a sudden change in the solvent conditions.

A closely related problem of buckling of an elastic filament in a viscous medium under uniaxial compression with fixed ends has been studied by Golubovic et al. [3]. It was found that the compression causes a transverse buckling instability with a characteristic wavelength. Through buckling, the compressional strain is reduced by expansion in the transverse direction at the expense of incurring bending deformation. The buckling phenomenon occurs spontaneously through a symmetry breaking, and the buckled configuration evolves as a phase ordering process.

The expansion of an elastic filament with free ends after a sudden change of solvent condition can exhibit a similar buckling instability to the case of applied external stress with fixed ends. This conclusion, however, is not obvious, due to the fact that whereas transverse buckling is the only mechanism to relieve the compressional strain in the case of fixed ends, a rod with free ends has the option of increasing its length through longitudinal expansion. We will identify the conditions under which longitudinal relaxation dominates the expansion process. Since free expansion through transverse buckling represents a richer and more interesting phenomenon, it will be the focus of our study. Nevertheless, longitudinal motion will be shown to have a significant effect on the dynamics, particularly on the expansion of the major axis of the rod.

We study the expansion dynamics by computer simulation and by extending previous analyses of the buckling phenomenon of filaments with fixed ends. We focus on the threedimensional structure of the dynamic process of expansion. The initial instability produces wavelike deformations in the two transverse directions. As the wavelike buckles grow, they coalesce to form helical structures in order to assume a growing conformation of constant curvature along the backbone. Domains of helical orientation spontaneously form due to statistical deviation of the buckle wavelength from the most unstable value. The sizes of the helical domains grow as the rod expands, until the rod conformation is dominated by a single helical orientation. The final structure is a pure helix (single handedness); although the system initially lacks any chiral preference. An interesting consequence of the growth of the helical domains is the phenomenon of selfpropulsion [4] of the expanding body throughout the expansion process. We present methods for measuring the average persistence length of the helical domains and explain their growth as a dissipative process.

\section{ELASTIC ROD MODEL}

We consider a thin rod of initial equilibrium length $L_{0}$ and uniform circular cross-sectional area $A_{0}$, made of isotropic elastic material. At the order of linear elasticity, the deformation of the rod can be decomposed into compression/ 
expansion, bending, and twist. In this first study, we ignore the twist deformation. In the case of a rod with free ends, twist deformation is dissipated out of the unconstrained body. The resistance to twist dissipation is much smaller than the resistance to drift motion of the entire rod; therefore, we neglect body rotation and focus on the drift motion of the chain. The shape of this thin rod is then described by a space curve $\mathbf{r}(s)$ where $s$ is an internal contour coordinate that runs from 0 to $L_{0}$. The linear compression/expansion strain, measured with respect to the initial rest length $L_{0}$, is given by

$$
e(s)=1-\left|\frac{\partial \mathbf{r}}{\partial s}\right|
$$

where we have defined the strain to be positive if it corresponds to compression. However, we are interested in the dynamic evolution of the rod after a sudden change in the solvent condition causes a change in the equilibrium length. Under the charged condition, the equilibrium length of the rod becomes $L_{f}>L_{0}$. The rod at the initial length $L_{0}$ will now experience a compressional strain with respect to the new equilibrium length. It is therefore more convenient to define a strain with respect to the new equilibrium length $L_{f}$,

$$
e(s)=\gamma_{0}-\left|\frac{\partial \mathbf{r}}{\partial s}\right|,
$$

where $\gamma_{0} \equiv L_{f} / L_{0}$ is the swelling ratio in the longitudinal direction.

The strain energy due to compression is then

$$
U_{c o m}=\frac{\tilde{\kappa}}{2} \int_{0}^{L_{0}} e(s)^{2} d s,
$$

where $\tilde{\kappa}$ is the compression modulus and is related to the Young's modulus $E$ of the elastic material by $\tilde{\kappa}=E A_{0}$. We will ignore the change in the Young's modulus due to the expansion of the rod.

The bending energy is due to curvature distortion of the rod from its equilibrium value, which in our case is taken to be zero. For an unstretchable rod model, such as the KratkyPorod wormlike chain model $[5,6]$, the curvature is simply $\partial^{2} \mathbf{r} / \partial s^{2}$. However, in our case the length of the rod is not conserved, and curvature is more properly defined as $(1 / \gamma) \partial / \partial s[(1 / \gamma)(\partial \mathbf{r} / \partial s)]$ where $\gamma(s)=(d \mathbf{r} / d s \cdot d \mathbf{r} / d s)^{1 / 2}$ relates the true arc length to the backbone internal coordinate. The bending energy is then given by

$$
U_{\text {bend }}=\frac{\tilde{\boldsymbol{\epsilon}}}{2} \int_{0}^{L_{0}}\left(\frac{1}{\gamma} \frac{\partial}{\partial s} \frac{1}{\gamma} \frac{\partial \mathbf{r}}{\partial s}\right)^{2} d s,
$$

where the bending modulus $\tilde{\epsilon}$ is found from the geometry of a gently bent cylinder to be $E A_{0}^{2} /(4 \pi)$ [7].

To numerically solve the dynamic equation of motion, we discretize the rod into $N+1$ "beads" with an initial bead separation of $l_{0}=L_{0} / N$. In this discretized representation, the compression and bending energies become

$$
U_{\text {com }}=\frac{\kappa}{2} \sum_{n=1}^{N} e_{n}^{2}
$$

where $e_{n}=\gamma_{0}-\left(\left|\mathbf{R}_{n+1}-\mathbf{R}_{n}\right| l_{0}\right)$ gives the internal strain, and

$$
U_{\text {bend }}=\frac{\epsilon}{2} \sum_{n=1}^{N-1}\left(\mathbf{t}_{n+1}-\mathbf{t}_{n}\right)^{2},
$$

where $\mathbf{t}_{n}=\left(\mathbf{R}_{n+1}-\mathbf{R}_{n}\right) /\left|\mathbf{R}_{n+1}-\mathbf{R}_{n}\right|$ is the unit tangent vector. The moduli are now given by $\kappa=l_{0} \tilde{\kappa}=E l_{0} A_{0}$ and $\epsilon$ $=\tilde{\epsilon} / l_{0}=E A_{0}^{2} /\left(4 \pi l_{0}\right)$ and have units of energy. We will use the moduli $\kappa$ and $\epsilon$ in the subsequent analysis.

We describe the dynamics of the thin rod by an overdamped Rouse dynamics in a viscous medium [8]. The equation of motion is

$$
\xi \frac{\partial \mathbf{R}_{n}(t)}{\partial t}=-\frac{\partial U}{\partial \mathbf{R}_{n}}+\mathbf{f}_{n}(t)
$$

where $\xi$ is the friction coefficient of the solvent and $\mathbf{f}_{n}(t)$ represents the random forces due to thermal noise. We will be interested in deformation energies (both bending and compression) that are large compared to the thermal energy; therefore, we will ignore any randomness except that associated with the initial perturbation. In Sec. IV, we further discuss the conditions where thermal fluctuations significantly contribute to the expansion dynamics and justify the neglect of the thermal fluctuations in our study.

Analysis of the equations of motion of the beads yields two fundamental time scales $\tau_{\text {com }}=\xi l_{0}^{2} / \kappa$ and $\tau_{\text {bend }}=\xi l_{0}^{2} / \epsilon$, associated with the location compression and bending relaxation, respectively. An additional time scale $\tau_{0}$ associated with the buckling phenomenon (see Sec. IV) arises from the expansion dynamics. In order to resolve these fundamental time scales $\left(\tau_{\text {com }}, \tau_{\text {bend }}\right.$, and $\left.\tau_{0}\right)$, the numerical solution of Eq. (7) is conducted using timesteps that are small in comparison. For the parameters that we choose, these three time scales are on the same order of magnitude; therefore, we arbitrarily choose to scale time by the fundamental buckling time $\tau_{0}$.

\section{EXPANSION DYNAMICS}

Our simulations focus on the dynamics of an initially straight rod of length $199.0 l_{0}$ expanding to a final equilibrium length of $298.5 l_{0}$ (50\% expansion). We use a Young's modulus of $10^{4} \mathrm{Nm}^{-2}$, representing that of polyethyleneglycol (400) methacrylate hydrogel [9]. Choosing an initial interbead spacing $l_{0}=346.38 \mathrm{~nm}$ and radius $R=292.40 \mathrm{~nm}$ results in a compression modulus $\kappa=9.304 \times 10^{-16} \mathrm{~J}$ and bending modulus $\epsilon=1.657 \times 10^{-16} \mathrm{~J}$.

We seed the initially straight chain with a small random perturbation in the transverse direction of an amplitude of $0.01 l_{0}$. (This initial condition does not reflect the tendency for a chain under thermal fluctuations to favor long wavelength perturbations. We have conducted simulations with perturbations produced by thermal equilibration of a chain with the initial interbead spacing, and observe qualitatively 

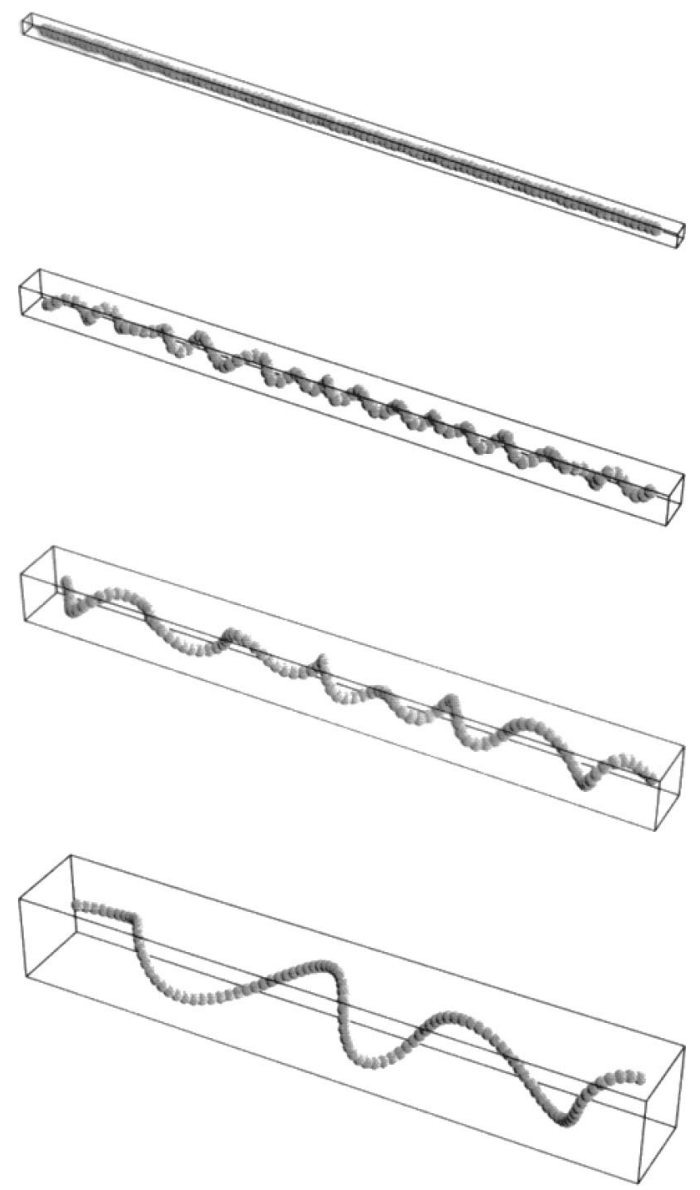

FIG. 1. Snapshots of the chain conformation during the free expansion process (100 out of the total 200 beads are shown). We show conformations at $3.94 \tau_{0}, 39.4 \tau_{0}, 394 \tau_{0}$, and $3940 \tau_{0}$, successively.

similar results to those with random perturbations.) At $t=0$, the equilibrium interbead separation is suddenly changed from $l_{0}$ to $1.5 l_{0}$, and the subsequent evolution of the structure of the chain is followed by numerically integrating Eq. (7).

We analyze the dynamics of the expanding chain using several metrics. The compression and bending energies are calculated from the bead coordinates using Eqs. (5) and (6). The shape of the chain is characterized by the radius-ofgyration tensor $\mathbf{T}$ [10]. The transverse buckling wavelength is measured by using the slope-slope correlation function $K_{s s}(r, t)$ [3]. And finally, the correlation length of the handedness of the transverse fluctuation is measured by introducing the torsion-torsion correlation function $K_{\tau \tau}(r, t)$.

A typical set of snapshots of the expanding chain is shown in Fig. 1. It is clearly seen that an initially straight chain develops buckling waves and that these waves grow through a coarsening process. The organization of the buckling waves into helices can also be observed.

Upon the development of buckling waves, the compression energy due to the altered equilibrium interbead spacing is partially converted into bending energy. This repartitioning of energy continues throughout the entire coarsening process. In Fig. 2, we show the evolution of the two energy contribu-

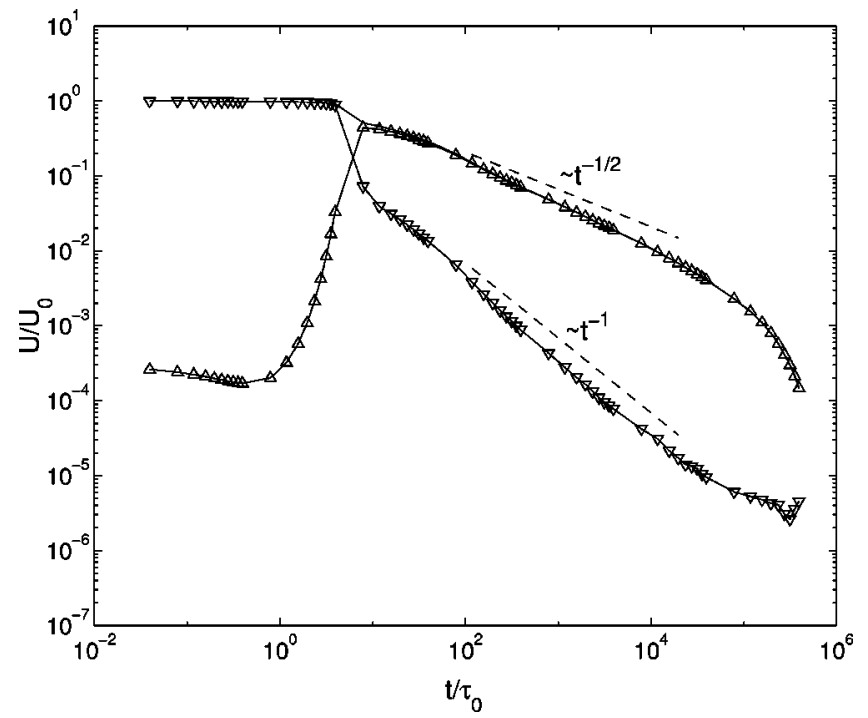

FIG. 2. Bending energy $U_{\text {bend }}(\triangle)$, compression energy $U_{\text {com }}$ $(\nabla)$, and total potential energy $U=U_{\text {bend }}+U_{\text {com }}$ during the expansion process. The total energy becomes indistinguishable from $U_{\text {bend }}$ for $t / \tau_{0}>5$.

tions during the expansion of the chain. The drop in the compression energy and sharp rise in the bending energy corresponds to a time when the buckling waves begin to grow significantly. Once the buckling waves are fully developed, both energy contributions decay due to dissipation. Over a broad intermediate time range, the decay of both contributions follows a power law with exponent $-1 / 2$ and -1 for the bending and compression energies, respectively. These features are qualitatively similar to those in the buckling dynamics due to compression along a rod with fixed ends, as analyzed in Ref. [3]. The very long time behavior is the relaxation of a bend with a length scale the size of the entire chain; relaxation of this long wavelength bend should be exponential and will be governed by the longest relaxation time associated with the bending motion. We note that full relaxation cannot be reached in the case of chains with fixed ends, and the simulation time in Ref. [3] was not long enough to reach this terminal regime.

To analyze the shape evolution of the expanding chain, we introduce the radius-of-gyration tensor $\mathbf{T}$, calculated from the bead coordinates $\mathbf{R}_{n}$ and the center-of-mass coordinate $\mathbf{R}^{c}(t)=\sum_{n=1}^{N+1} \mathbf{R}_{n}(t) /(N+1)$ :

$$
T_{i j}(t)=\frac{1}{N+1} \sum_{n=1}^{N+1}\left[R_{i, n}(t)-R_{i}^{c}(t)\right]\left[R_{j, n}(t)-R_{j}^{c}(t)\right]
$$

where $i$ and $j$ denote cartesian components $x, y$, and $z$, and $n$ denotes the discrete backbone coordinate. The square roots of the three eigenvalues of the tensor $\mathbf{T}\left(R_{1}, R_{2}\right.$, and $\left.R_{3}\right)$ give a measure of the size of the chain along the major axis $\mathbf{x}_{1}$ and the two minor axes $\mathbf{x}_{2}$ and $\mathbf{x}_{3}$, which are the eigenvectors of T. Figure 3 shows the evolution of the three principal radii of gyration with time. Since the major radius $R_{1}$ starts from a large nonzero initial value, we present data for $R_{1}(t)-R_{1}(0)$. This difference is seen to increase linearly at 

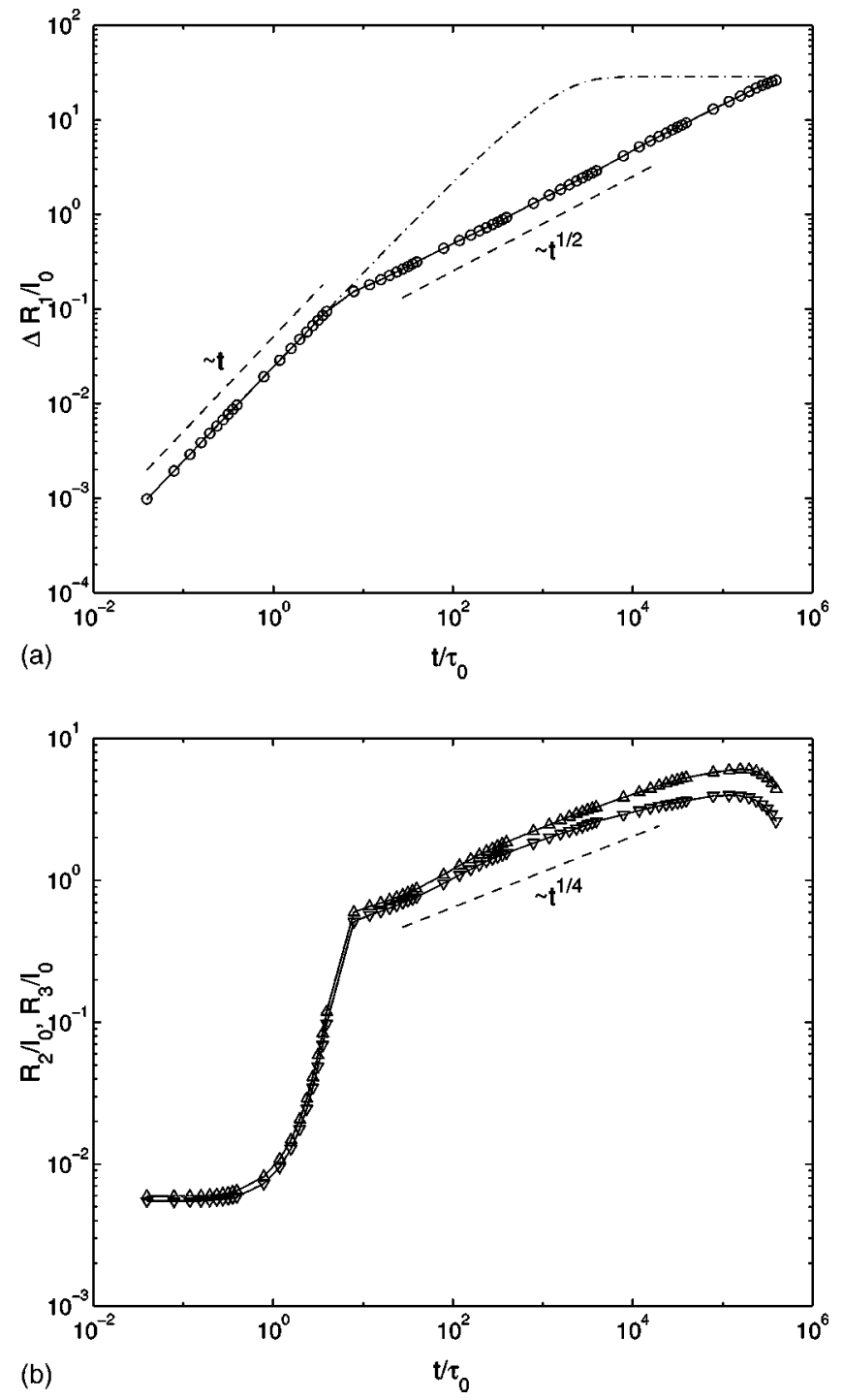

FIG. 3. (a) Expansion dynamics of the major axis $\Delta R_{1}=R_{1}$ $-R_{1}(t=0)$. The dashed-dotted curve corresponds to the analytical solution [Eq. (16)] for the expansion of a rod in one dimension. (b) Time evolution of the minor axes $R_{2}(\triangle)$ and $R_{3}(\nabla)$ during the expansion process.

first and then turn into a slower square-root power increase around the time the buckling waves develop. The two minor radii start at a small value and then rapidly increase when the buckling waves begin to develop. Afterwards, they follow a $t^{1 / 4}$ power law growth until eventually dropping back to zero when the chain is fully relaxed. The small differences in $R_{2}$ and $R_{3}$ are due to statistical errors, as these two axes are equivalent.

Following Ref. [3], we define the slope-slope correlation function $K_{s s}$ to calculate the characteristic wavelength $\lambda$ of the buckling waves. The transverse slope $\mathbf{V}_{n}^{T}$ is the projection of the relative bead position $\mathbf{V}_{n}=\mathbf{R}_{n+1}-\mathbf{R}_{n}$ onto the tranverse axes $\mathbf{x}_{2}$ and $\mathbf{x}_{3}$

$$
\mathbf{V}_{n}^{T}(t)=\left[\mathbf{x}_{2}(t) \cdot \mathbf{V}_{n}(t)\right] \mathbf{x}_{2}+\left[\mathbf{x}_{3}(t) \cdot \mathbf{V}_{n}(t)\right] \mathbf{x}_{3},
$$

and the slope-slope correlation function is defined as

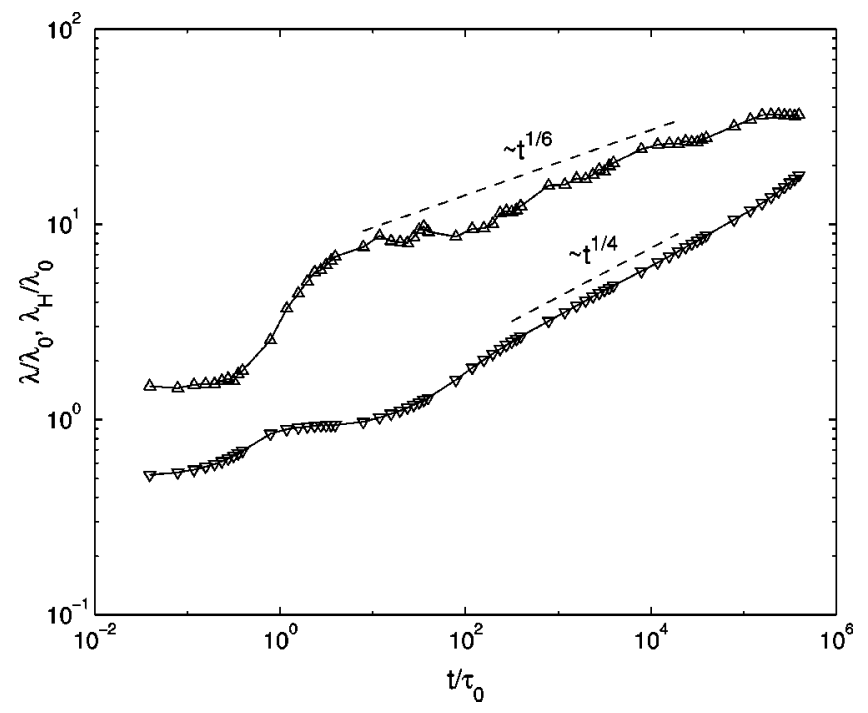

FIG. 4. The time evolution of the average wavelength of the buckled perturbation $\lambda(\nabla)$ and the average persistence length of the buckle handedness $\lambda_{H}(\triangle)$.

$$
K_{s s}(r, t)=\left\langle\mathbf{V}_{n+r}^{T}(t) \cdot \mathbf{V}_{n}^{T}(t)\right\rangle,
$$

where the angular brackets refer to a statistical averaging of many runs with different realizations of the initial random seeding. For a perfect sinusoidal wave, the function $K_{s s}(r, t)$ changes sign twice during one full period in $r$, the first zero occurring at one-quarter of the wavelength $\lambda$. We therefore define the characteristic wavelength as four times the value of $r$ where $K_{s s}(r, t)$ first becomes zero.

In order to measure the persistence of the handedness of the transverse fluctuation $\lambda_{H}$, we introduce the torsiontorsion correlation function $K_{\tau \tau}$. For a space curve, torsion $\tau$ is defined to measure the instantaneous amount that the curve is distorted out of a planar path. Mathematically [11],

$$
\tau=-\mathbf{n} \cdot \frac{d \mathbf{b}}{d s},
$$

where $\mathbf{n}$ is the unit normal, which is a measure of the normalized rate of change of the tangent vector $\mathbf{t}$ with respect to the arc length $s$ and $\mathbf{b}$ is the unit binormal, defined as the cross product of the tangent vector with the unit normal. The sign of the torsion determines the handedness of the distortion: a right handed distortion generates a positive torsion; a left handed distortion generates a negative torsion. The torsion-torsion correlation function is defined as

$$
K_{\tau \tau}(r, t)=\left\langle\tau_{n+r}(t) \tau_{n}(t)\right\rangle
$$

This function switches sign at half the correlation length of the helical orientation $\lambda_{H}$.

In Fig. 4, we show the time evolution of the buckling wavelength $\lambda$ and the helical correlation length $\lambda_{H}$. The initial random seeding does not favor any particular wavelength. However, after some initial incubation period, the transverse fluctuation picks up a dominant wave number corresponding to a plateau in $\lambda(t)$. Subsequently, the coarsening 


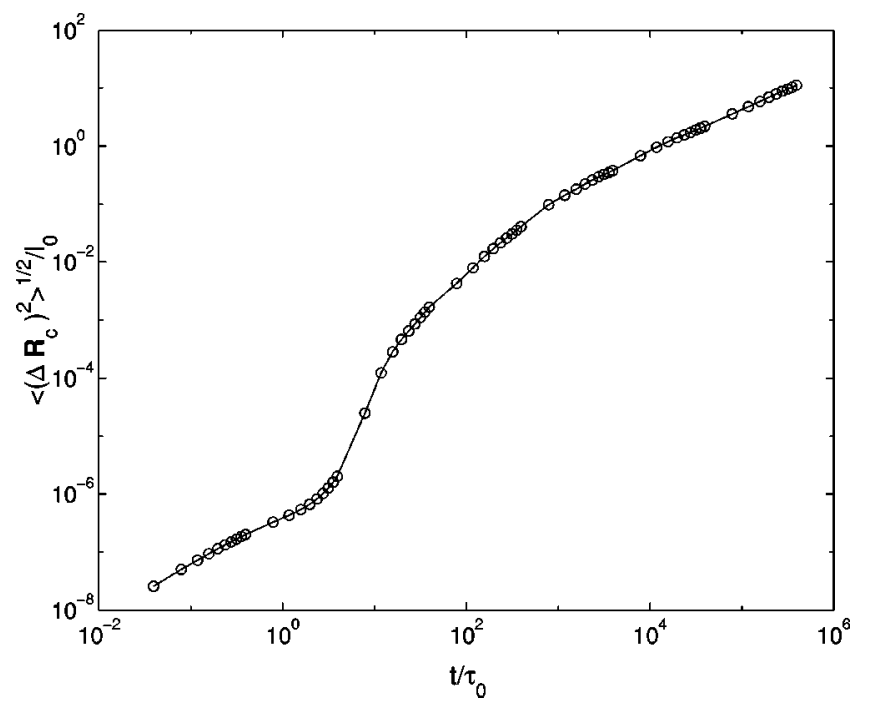

FIG. 5. Time evolution of the center-of-mass displacement $\left(\left\langle\left(\Delta \mathbf{R}_{c}\right)^{2}\right\rangle^{1 / 2}=\left\langle\left[\mathbf{R}_{c}-\mathbf{R}_{c}(t=0)\right]^{2}\right\rangle^{1 / 2}\right)$ during the expansion process.

of the buckles leads to an increase in the average wavelength, manifested as a $t^{1 / 4}$ power law for a broad range of the intermediate times. The growing wavelike buckles in three dimensions organize into helical domains, which are separated by domain boundaries that may be viewed as imperfections within the helical structure. The energy associated with the boundary imperfections is relaxed by diffusion of the imperfections from the system, thus the helical correlation length increases until the structure is a pure helix.

The development of helices generates a drift motion in the center-of-mass displacement of the expanding structure. The rate of self-propulsion depends on the structure of the expanding body, the rate of relaxation, and the net amount of helicity in the chain. Figure 5 shows the displacement of the center of mass during the expansion process. The dynamics of the motion is clearly rather complex. In particular, accelerated motion takes place during the rapid development of the buckling waves. The late stage motion appears to be linear and is probably due to the self-propulsion generated during the relaxation of the one single helix.

In order to clearly show the formation of a helical buckled conformation, we have performed simulations where the initial condition is seeded with a preferential perturbation along one of the two transverse directions. Specifically, we set the $y$ component of the initial transverse perturbation to a sinusoidal wave with an amplitude of $0.1 l_{0}$ and with a wavelength $\lambda_{0}$, the fastest growing wavelength determined from the linear stability analysis (see next section). The $z$ component of the initial perturbation is set to a random fluctuation with an amplitude of $0.01 l_{0}$. The time evolution of the two minor axes is shown in Fig. 6. The separation of the two minor axes $R_{2}$ and $R_{3}$ at the beginning of the expansion process reflects the favoring of the $y$ component. The two magnitudes increase parallel to each other until $R_{2}$ has expanded to a threshold value, at which point $R_{2}$ levels off. The smaller minor axis $R_{3}$ catches up to the larger minor axis $R_{2}$. Thereafter, they continue to increase; however, the

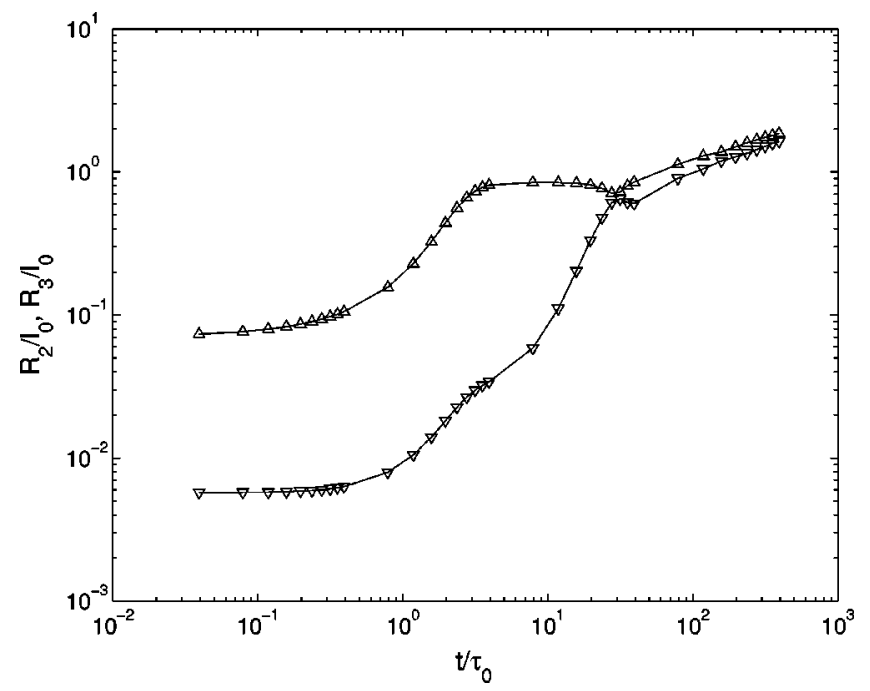

FIG. 6. Early stage time evolution of the minor axes $R_{2}(\triangle)$ and $R_{3}(\nabla)$. This simulation seeds one transverse direction with the unstable wavelength $\lambda_{0}$ of amplitude $0.1 l_{0}$ and the other transverse direction with random perturbation of amplitude $0.01 l_{0}$.

two radii become roughly equal. The structure at this point becomes clearly helical, as confirmed by direct visualization of the chain configuration. The helical conformation minimizes the bending energy by distributing a constant curvature over the length of the elastic chain.

\section{LINEAR STABILITY ANALYSIS AND DYNAMIC SCALING}

In this section, we perform a linear stability and scaling analysis with the goal of obtaining a physical understanding of the results presented in the previous section. We first analyze the early stage dynamics that focuses on the initial expansion of the chain in the longitudinal direction and the development of the buckling wave. We then use a simple energy argument to understand the driving force for the formation of helices. Finally, we use scaling analysis to rationalize the observed intermediate and long time scaling behavior of the various quantities.

At the early stage of the expansion, the chain is essentially straight with small transverse perturbations. It is therefore convenient to decompose the chain configuration into longitudinal and transverse components, with the longitudinal axis parallel to the initial orientation of the chain. We define a longitudinal deformation $u(s)$ through $\mathbf{r}_{L}=[s$ $+u(s, t)] \hat{x}$, where $\mathbf{r}_{L}$ is the longitudinal position of the chain backbone at internal coordinate $s$. Note here that the longitudinal deformation is defined with respect to the initial equilibrium position of the chain. The transverse deformation $\mathbf{R}_{T}(s)$ at $s$ is simply the deviation from the initial chain axis. The equations of motion for these components are obtained in a manner similar to Eq. (7) taking special care to maintain the dimensions in the continuous model. They are

$$
\xi \frac{\partial u}{\partial t}=-\kappa \frac{\partial e}{\partial s}-\epsilon l_{0}^{2} \frac{\partial}{\partial s}\left\{\frac{1}{\gamma} \frac{\partial}{\partial s}\left[\frac{1}{\gamma^{2}} \frac{\partial}{\partial s}\left(\frac{1}{\gamma} \frac{\partial(s+u)}{\partial s}\right)\right]\right\}
$$


$\xi \frac{\partial \mathbf{R}_{T}}{\partial t}=-\kappa \frac{\partial}{\partial s}\left(e \frac{\partial \mathbf{R}_{T}}{\partial s}\right)-\epsilon l_{0}^{2} \frac{\partial}{\partial s}\left\{\frac{1}{\gamma} \frac{\partial}{\partial s}\left[\frac{1}{\gamma^{2}} \frac{\partial}{\partial s}\left(\frac{1}{\gamma} \frac{\partial \mathbf{R}_{T}}{\partial s}\right)\right]\right\}$

The early stage dynamics is dominated by the compression energy. Since the initial conformation is essentially straight, the beads move in the longitudinal direction as if they are confined to move in one dimension. Ignoring the transverse components leads to the following simplified equation of motion for the longitudinal deformation

$$
\xi \frac{\partial u}{\partial t}=\kappa \frac{\partial^{2} u}{\partial s^{2}}
$$

It is convenient to define the internal coordinate $s$ $\in\left[-L_{0} / 2, L_{0} / 2\right]$. This equation is supplemented by the final value $u(s, \infty)=\left(\gamma_{0}-1\right) s=e_{0} s$ and the zero strain boundary conditions at the two chain ends. The solution can be readily obtained as an eigenfunction expansion that reads

$$
\begin{aligned}
u= & e_{0} s-\frac{e_{0} L_{0}}{\pi^{2}} \sum_{p=0}^{\infty} \frac{(-1)^{p}}{\left(p+\frac{1}{2}\right)^{2}} \sin \left[2 \pi\left(p+\frac{1}{2}\right) \frac{s}{L_{0}}\right] \\
& \times \exp \left[-4 \pi^{2} \kappa\left(p+\frac{1}{2}\right)^{2} \frac{t}{\xi L_{0}^{2}}\right] .
\end{aligned}
$$

Equation (16) defines a set of relaxation times for the eigenmodes. In particular, a "Rouse-like" time associated with the slowest longitudinal relaxation mode can be identified as $\tau_{\text {long }}=\xi L_{0}^{2} /\left(\pi^{2} \kappa\right)$.

Using Eq. (16), we obtain the evolution in the radius of gyration for a chain expanding in one dimension. For short times, the change in the radius of gyration is given by

$$
\begin{aligned}
R_{g}-R_{g}(t=0) & =\sqrt{\frac{1}{L_{0}} \int_{-L_{0} / 2}^{L_{0} / 2}(s+u)^{2} d s}-\frac{L_{0}}{2 \sqrt{3}} \\
& \approx \frac{2 \sqrt{3} \kappa e_{0}}{\xi L_{0}} t \\
& =L_{0} e_{0} \frac{2 \sqrt{3}}{\pi^{2}} \frac{t}{\tau_{\text {long }}} .
\end{aligned}
$$

This predicted linear behavior is in perfect agreement with the simulation result shown in Fig. 3.

The one-dimensional configuration of a chain with internal compressional strain is unstable with respect to small transverse perturbation. The initial instability can be understood through a simple linear stability analysis. Representing the transverse perturbation as a sinusoidal wave with wave number $k, \mathbf{R}_{T} \sim \mathbf{A}_{k} \exp (i k s)$, and substituting it into the equation of motion, Eq. (14), we find that the amplitude of the perturbation $\mathbf{A}_{k}$ will grow for $k$ 's in the range $|k|$ $<e_{0}^{1 / 2}(\kappa / \epsilon)^{1 / 2} / l_{0}$. The most unstable (i.e., fastest growing) mode is that with $k_{0}= \pm e_{0}^{1 / 2}(\kappa / \epsilon)^{1 / 2} /\left(\sqrt{2} l_{0}\right)$, corresponding to a wavelength $\lambda_{0}$ of $2 \pi l_{0}\left[\kappa e_{0} /(2 \epsilon)\right]^{-1 / 2}$. The most unstable mode predicted from the linear stability analysis does not favor the $y$ or $z$ directions, nor does it favor the cosine or sine contributions. This suggests that the transverse perturbations in the two unexplored dimensions initially are independent of each other. However, statistically, the amplitude of the most unstable mode is equivalent in these two dimensions.

The linear stability analysis predicts an exponential growth of the most unstable mode at a rate of $\tau_{0}^{-1}$ $=\kappa^{2} e_{0}^{2} /\left(4 l_{0}^{2} \xi \epsilon\right)$, thus the transverse displacement becomes significant when $t \sim \tau_{0}$. This time therefore signals the termination of the one-dimensional growth dominated by the compression energy. This is shown clearly as the end of the plateau in the compression energy in Fig. 2, and the deviation from linear growth in the radius of gyration of the major axis (Fig. 3). This time also correlates well with the onset of scaling behavior in other properties.

The expanding transverse buckles force the bending energy to overcome the compression energy at times $t>\tau_{0}$. The growing wavelike structure locally conforms to a path between a planar wave and a helix. The three-dimensional buckled structure relaxes by following the steepest descent on the potential energy surface. We now show that the bending energy favors organization of the buckles into helices. To this end, we focus on the bending energy contribution in a segment of the chain spanning a single period of wavelength $\lambda_{0}$. Since the longitudinal expansion requires an orchestrated motion of the entire chain, the transverse buckles grow outward without appreciably spreading longitudinally. Thus, for the present purpose we keep the longitudinal distance of this chain segment fixed, so that chain expansion occurs only through the growth of the buckling wave in the transverse directions. We represent the distortion of the chain by assuming

$$
\mathbf{r}=X(s) \hat{x}+\Delta r_{y} \sin \left(k_{0} s\right) \hat{y}+\Delta r_{z} \cos \left(k_{0} s\right) \hat{z},
$$

subject to a given swelling ratio $|d \mathbf{r} / d s|=\gamma$, with $\gamma$ varying between 1 and 1.5 corresponding to the initial and final length of the chain, respectively. Note that either the sine or cosine part in the above equation corresponds to a twodimensional wave, whereas when both sine and cosine are present with equal coefficients, we obtain a perfect helix. Therefore, we plot the bending energy as a function of $\left[\Delta r_{y}-\Delta r_{z}\right] / \lambda_{0}$; the result is shown in Fig. 7. It is clear that the helical structure is an energy minimum at all values of $\gamma$ (representing a time progression in the expansion), reflecting the tendency of the wavelike structure to assume a conformation of constant curvature. The energy difference between the helix and a two-dimensional deformation increases with increasing swelling ratio and approaches zero as the swelling ratio approaches one, the latter being consistent with the lack of a transverse directional preference of the buckling predicted by the linear stability analysis. Thus the formation of helices is a nonlinear effect.

As the wavelike deformations grow in the transverse direction, the driving force for coalescing into a helical conformation increases. The handedness of a helix is dictated by 


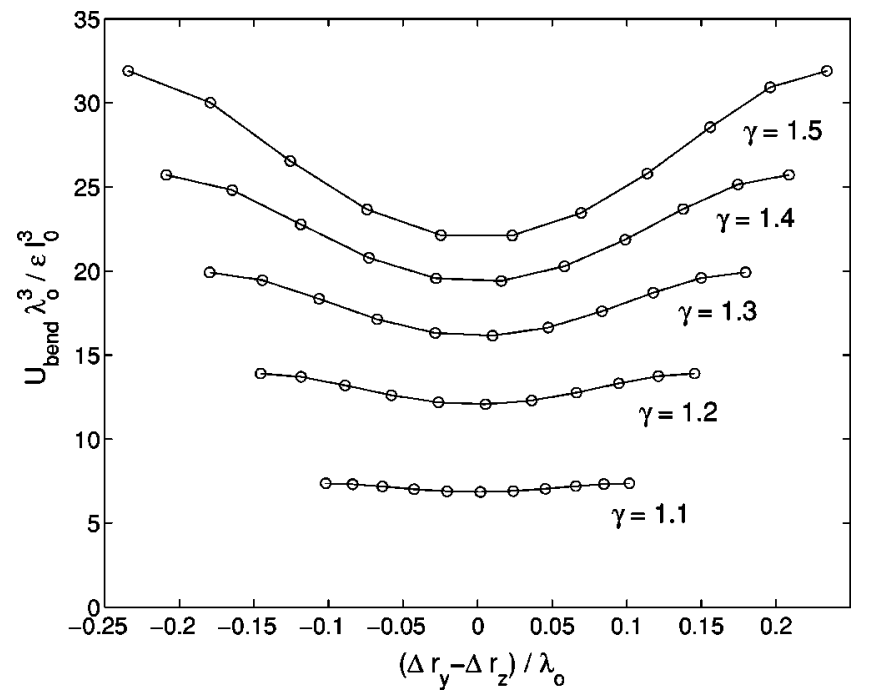

FIG. 7. Bending energy for a single period wavelike distortion. The amplitudes $\Delta r_{y}$ and $\Delta r_{z}$ correspond to the relative contribution of the sine component and the cosine component respectively.

the sign of the local torsion $\tau$. The random initial perturbation does not favor one over the other. However, because of the continuity of the space curve, correlation develops as the chain expands so that the local torsion persists over a length $\lambda_{H}$. The result is a helical structure with helical domains of length $\lambda_{H}$, which are separated by kink domain boundaries.

The dynamics for the intermediate times $\tau_{f}>t>\tau_{0}$, where $\tau_{f}$ is a terminal relaxation time scale to be obtained later, is characterized by power law dependence in a number of properties. To understand the observed scaling behavior, we perform a scaling analysis, following similar arguments given in Refs. $[3,12]$ in the study of compressed rods and membranes.

We choose a transverse displacement scale $R_{T}$, ignoring for the present purpose the difference between $R_{2}$ and $R_{3}$. The longitudinal deformation is characterized by the increase of the length along the major axis $\Delta R_{1}$, which scales similarly to the displacement $u$ introduced at the beginning of this section. The length scale for the bending deformation is the wavelength of the buckles $\lambda$ and that for the longitudinal displacement is $L_{0}$. The fact that the contour length increases will be accounted for by the leading dependence in longitudinal strain, but otherwise has little effect.

We start with the scaling behavior for the buckling wavelength $\lambda$. The driving force for buckling is due to both the compression energy and the bending energy. Assuming that the two driving forces contribute roughly equally, we have, from the transverse equation of motion [Eq. (14)],

$$
\xi \frac{R_{T}}{t} \sim \kappa \frac{e R_{T}}{\lambda^{2}} \sim \epsilon l_{0}^{2} \frac{R_{T}}{\lambda^{4}} .
$$

The above relation can be rewritten using the initial strain $e_{0} \equiv \gamma_{0}-1$, and the wavelength $\lambda_{0}$ and time scale $\tau_{0}$ $=4 l_{0}^{2} \xi \epsilon /\left[\kappa^{2} e_{0}^{2}\right]$ identified from the linear stability analysis, as

$$
\frac{\tau_{0} R_{T}}{t} \sim \frac{e R_{T} \lambda_{0}^{2}}{e_{0} \lambda^{2}} \sim \frac{R_{T} \lambda_{0}^{4}}{\lambda^{4}} .
$$

It can be readily seen that the buckling wavelength scales as $\lambda \sim \lambda_{0}\left(t / \tau_{0}\right)^{1 / 4}$. The internal strain is seen to scale as $e$ $\sim e_{0}\left(t / \tau_{0}\right)^{-1 / 2}$. Substituting the internal strain into the compression energy yields $U_{\text {com }} \sim \kappa\left(L_{0} / l_{0}\right) e_{0}^{2}\left(t / \tau_{0}\right)^{-1}$.

To understand the scaling behavior of the increase in the length along the major axis of the rod, we analyze the governing equation for the longitudinal motion [Eq. (13)]. This analysis requires careful consideration of the appropriate length scales. The contribution of the compression energy is relatively unaffected by the presence of transverse buckles; therefore, the proper backbone length scale for the compression energy is $L_{0}$, leading to

$$
\xi \frac{\Delta R_{1}}{t} \sim \kappa \frac{e}{L_{0}} .
$$

Using the scaling for the internal strain $e$, we obtain the scaling for the longitudinal displacement $u$ as

$$
\Delta R_{1} \sim \kappa \frac{e_{0} t^{1 / 2} \tau_{0}^{1 / 2}}{\xi L_{0}} \sim\left(\frac{t}{\tau_{\text {bend }}}\right)^{1 / 2} \frac{l_{0}^{2}}{L_{0}},
$$

where the last expression results from using the expressions for $\tau_{0}$ and $\tau_{\text {bend }}=\xi l_{0}^{2} / \epsilon$. This accounts for the observed $t^{1 / 2}$ power law increase in the major axis shown in Fig. 3.

The scaling behavior for $R_{T}$ shown in Fig. 3 can be understood by going back to the expression for the internal strain that consists of both longitudinal and transverse contributions

$$
e=e_{0}-\frac{\partial u}{\partial n}-\frac{1}{2}\left(\frac{\partial \mathbf{R}_{T}}{\partial n}\right)^{2} \sim e_{0}-\frac{\Delta R_{1}}{L_{0}}-\frac{R_{T}^{2}}{\lambda^{2}} .
$$

For $t>\tau_{0}$ the strain becomes negligible compared to each individual term on the right hand side of the equation. Thus, we can obtain the scaling behavior for $R_{T}$ by balancing the three terms on the right. Assuming for the moment that we can ignore the $\Delta R_{1} / L_{0}$ term, we have

$$
R_{T} \sim e_{0}^{1 / 2} \lambda \sim e_{0}^{1 / 2} \lambda_{0}\left(\frac{t}{\tau_{0}}\right)^{1 / 4}
$$

which is in agreement with the power law behavior in Fig. 3. Combining the scaling for $R_{T}$ and $\lambda$, we obtain the scaling for the bending energy $U_{\text {bend }} \sim \epsilon l_{0} L_{0} R_{T}^{2} / \lambda^{4}$ $\sim \kappa e_{0}^{2}\left(L_{0} / l_{0}\right)\left(t / \tau_{0}\right)^{-1 / 2}$. The power law behavior of $R_{T}$ [Eq. (24)] terminates when the second term in Eq. (23) $\Delta R_{1} / L_{0}$ is no longer negligible, and this happens when

$$
\Delta R_{1} \sim e_{0} L_{0}
$$

or

$$
t \sim \tau_{f} \sim \tau_{\text {bend }} e_{0}^{2}\left(\frac{L_{0}}{l_{0}}\right)^{4}
$$


$\tau_{f}$ thus defines the terminal relaxation time when the rod has approached its final equilibrium length. This terminal relaxation time can also be obtained as the time required for the wavelength of the buckles to reach the full length of the rod. Setting $\lambda \sim L_{0}$, we have

$$
\tau_{f} \sim \tau_{\text {bend }}\left(\frac{L_{0}}{l_{0}}\right)^{4},
$$

which apart from some numerical factor is identical to the scaling expression Eq. (26). We note that this relaxation time is nothing but the transverse relaxation time for a semiflexible polymer $[13,14]$. Interestingly, the relaxation time associated with the longitudinal relaxation [Eq. (16)] does not play an explicit role in our study. This is because buckling appears long before this relaxation time is reached since

$$
\frac{\tau_{0}}{\tau_{\text {long }}} \sim \frac{4 \epsilon l_{0}^{2}}{\kappa e_{0}^{2} L_{0}^{2}} \ll 1 .
$$

The time scale $\tau_{\text {long }}$ becomes obliterated once buckling waves dominate the relaxation dynamics. This inequality in fact specifies the condition under which the free expansion problem exhibits similar transverse buckling to that in a compressed rod with fixed ends. This condition reflects the significantly larger resistance associated with longitudinal relaxation than that associated with transverse buckling. If the parameters are altered such that $\tau_{\text {long }} \sim \tau_{0}$, then even though the transverse buckling instability may still exists [15], the buckling waves will not be able to grow to any significant extent since the driving force for buckling will have been dissipated through longitudinal relaxation. For an elastic rod made of isotropic material, this will be the case if the initial strain is less or comparable to the inverse of the aspect ratio of the rod. (This result can be obtained by substituting the explicit dependence of the bending and compression moduli on the diameter of the rod and on the Young's modulus of the materials.) For long rods with large strain, the scenario we have presented in this study will prevail.

The increase of the helical persistence length $\lambda_{H}$ is necessarily slower than that of the wavelength $\lambda$. The wavelength $\lambda$ grows by two modes of relaxation. It grows internally at the helical domain boundaries; two helices meet and eliminate one another by turning the imperfection around the axis of orientation. This process increases the wavelength without effecting the length of the two helical domains. The wavelength also grows at the chain ends by turning the end helices around the axis of orientation. This process increases both the wavelength and the helical domain length. The helical persistence length grows at a slower rate than the wavelength because the helical domains grow only by diffusion of writhe out of the ends, and the wavelength grows by both internal relaxation and unwinding at the ends. The physics of the relaxation of two preexisting competing helical structures has recently been analyzed for a chain of fixed length and bending/twisting degrees of freedom [16]. Two types of imperfection front propagation are identified: "crankshafting," where one helix spins around the axis of the other at the chain end (end dissipation), and "speedometer-cable mo- tion,' where each helix revolves around their own axis (internal relaxation). These modes of front propagation are consistent with our description of domain relaxation; however, our problem is complicated by the existence of many domains of helicity, and these two modes occur simultaneously during the free expansion process. Also, the helices studied in Ref. [16] correspond to energy minima in a bistable system, while the helices appearing in our study are transient structures that arise spontaneously during the relaxation process in a system lacking an obvious propensity for helical formation. The apparent $t^{1 / 6}$ growth in $\lambda_{H}$ observed here is a result of a rather complex relaxation mechanism that remains to be investigated further.

Finally, we discuss the effects of neglecting thermal fluctuation in our study. To this end, we compare the thermal energy with the compression and bending energies. Since the compression energy decays faster than the bending energy, thermal fluctuation is expected to affect the system behavior when $U_{c o m} \sim N k_{B} T$. Since $U_{c o m} \sim \kappa N e_{0}^{2}\left(t / \tau_{0}\right)^{-1}$, the two energies become comparable when $t=\tau_{T} \sim\left(\kappa / k_{B} T\right) e_{0}^{2} \tau_{0}$. For our choice of parameters and assuming room temperature, $\kappa / k_{B} T \approx 6 N^{2}$; thus we estimate that $\tau_{T} \sim 10^{4} \tau_{0}$. On the other hand, the bending energy remains much larger than the thermal energy until well past the terminal relaxation time $\tau_{f}$. Thus the $t^{-1 / 2}$ decay in the bending energy shown in Fig. 2 will be unaffected by the thermal fluctuation, while the $t^{-1}$ decay in the compression energy will reach a plateau value corresponding to the thermal energy of the rod at $t \sim \tau_{T}$. However, since the scaling behavior of most of the properties studied in this work is determined by the bending energy, thermal fluctuation has little effect on these properties. Interestingly, we find from separate calculations that included Brownian forces, that even the $t^{1 / 2}$ growth in the length of the major axis (which is expected to be affected by the compression energy) persists well past the time when the compression energy reaches a plateau.

We note that there are many situations in which thermal fluctuation plays an essential role in the dynamics of a semiflexible polymer. Stress relaxation in semiflexible polymers is an example that has attracted considerable attention in recent years $[13,14,17,18]$. Similarly, the propagation of tension upon pulling a semiflexible polymer by one end is shown to depend crucially on the thermal fluctuation spectrum in the initial configuration [19]. While the inclusion of thermal fluctuation in our work would undoubtedly be more realistic and would lead to slight modifications in the behavior of some properties, we have chosen to focus our attention on situations where relaxation is driven by large mechanical energies and hence the omission of thermal fluctuation is justified.

\section{CONCLUSIONS}

The free expansion of an elastic filament progresses along the energetic path of least resistance, notably marked by the emergence of the transverse buckling, which occurs to distribute the energy between compression and bending. The wavelike buckles grow as a coarsening process until the wavelength reaches the full length of the chain, at which 
point the chain relaxes back to its equilibrium straight conformation. The buckles expand in the transverse direction and prefer to assume a helical orientation due to a nonlinear effect, which is not predicted by linear stability analysis. Although the energetically preferred conformation as the transverse displacement grows is a pure helix (single handedness), the local handedness is determined by the local torsion; therefore, the wavelike buckles coalesce into helical domains separated by kink imperfections, which are eliminated from the expanding body by diffusion from the chain ends. The helical domains grow until the relaxing conformation achieves a pure helical orientation, where the handedness is determined by the orientation of statistical dominance.

The relaxing helix undergoes self-propulsion, which is similar in nature to the propulsive motion of flagellated micro-organisms, as it strives to achieve the minimum energy, straight conformation. The existence of domains of handedness in the expanding structure extends the comparison between our problem and micro-organism propulsion, since some bacterial flagella employ competition between structures of opposite chirality to drive motion [16]. The dynamics of the drift motion of the entire chain is rather complicated due to the structural complexity of the relaxing body; therefore, a scaling analysis of the center-of-mass motion from the dynamic equations of motion would not prove effective. Furthermore, in the case of flagellated microorganisms, an important contributing factor toward the rate of self-propulsion is the effect of chain twisting on the hydrodynamic behavior of the elastic rod [4]. We have analyzed the structure of the freely expanding rod and predicted that self-propulsion is an interesting consequence of the higher order structure that is established during the relaxation process. However, to quantify the center-of-mass displacement, the additional twist degree of freedom should be included in the elastic rod model [20-22].

The expansion dynamics present an interesting problem of symmetry breaking where the system breaks symmetry in two ways. The straight rod spontaneously buckles in the transverse direction and the wavelike buckles further orga- nize into helical domains of common handedness. The former symmetry breaking occurs to preferentially distribute the energy between bending and compression, rather than dissipating the energy through compression alone. Energy redistribution during the relaxation process is common in problems of stress dissipation. Similar to expanding rods, the expansion of compressed membranes exhibits transverse buckling in order to alleviate the compression energy [12]. Similarly, when an elastic cube is stressed through inward pointing forces at the cube vertices, the cube buckles along the cube edges [23]. An initially twisted rod may unwind the twist density via "'geometric untwisting," where twist is converted to writhe, and stress is dissipated through drift motion of the chain backbone [20]. This process, in which energy is redistributed between twist and bending, is obviously analogous to the free expansion problem we study here. This mode of relaxation, however, is the dominant mechanism only when the moment about the twisted chain generated by the torsional deformation cannot overcome the rotational resistence. Thus there is a further similarity between the relaxation of twist deformation and the free expansion of elastic filaments, as in both systems redistribution of energy through instability occurs only under certain conditions.

A recent theoretical analysis of DNA condensation [24] suggests that the chain collapse occurs via an Euler buckling instability, thus partitioning the total energy into bending and electrostatic energy. Our analysis of the freely expanding rod provides insight into the existence of higher order structure within the buckle phase; therefore, if the chain collapse occurs through a similar instability, it is likely that the condensing DNA strand also contains helical domains. We are interested in analyzing the effect of helicity on the chain collapse dynamics and determining its role in the morphology of the condensed DNA strand.

\section{ACKNOWLEDGMENTS}

This work was supported in part by the National Science Foundation (DMR-9970589) and the Camille and Henry Dreyfus Foundation (TC-96-063).
[1] G.M. Eichenbaum, P.F. Kiser, S.A. Simon, and D. Needham, Macromolecules 31, 5084 (1998).

[2] E. Evans, H. Bowman, A. Leung, D. Needham, and D. Tirrell, Science 273, 933 (1996).

[3] L. Golubovic, D. Moldovan, and A. Peredera, Phys. Rev. E 61, 1703 (2000).

[4] T.Y.T. Wu, C.J. Brokaw, and C. Brennen, Swimming and Flying in Nature (Plenum, New York, 1975), Vol. 1.

[5] O. Kratky and G. Porod, Recl. Trav. Chim. Pays-Bas. 68, 1106 (1949).

[6] H. Yamakawa, Helical Wormlike Chains in Polymer Solutions (Springer-Verlag, Berlin, 1997).

[7] L.D. Landau and E.M. Lifshitz, Theory of Elasticity (Pergamon New York, 1986).

[8] M. Doi and S.F. Edwards, The Theory of Polymer Dynamics (Clarendon, Oxford, 1986).
[9] K. Murphy and N. Ravi, Polym. Prepr. (Am. Chem. Soc. Div. Polym. Chem.) 40, 630 (1999).

[10] J. Rudnick and G. Gaspari, Science 237, 384 (1987).

[11] R.C. Wrede, Introduction to Vector and Tensor Analysis (Dover, New York, 1972).

[12] L. Golubovic and D. Moldovan, Phys. Rev. Lett. 82, 2884 (1999).

[13] D.C. Morse, Phys. Rev. E 58, R1237 (1998).

[14] F. Gittes and F.C. MacKintosh, Phys. Rev. E 58, R1241 (1998).

[15] Note that the critical compressional strain for Euler buckling [7] is $e_{c} \sim \tilde{\boldsymbol{\epsilon}} \tilde{\kappa}^{-1} L^{-2}$, while the condition in Eq. (28) requires $e_{0} \gg \tilde{\epsilon}^{1 / 2} \tilde{\kappa}^{-1 / 2} L^{-1}$.

[16] R.E. Goldstein, A. Goriely, G. Huber, and C.W. Wolgemuth, Phys. Rev. Lett. 84, 1631 (2000). 
[17] P. Dimitrakopoulos, J.F. Brady, and Z.-G. Wang, Phys. Rev. E (to be published).

[18] R. Granek, J. Phys. II 7, 1761 (1997).

[19] U. Seifert, W. Wintz, and P. Nelson, Phys. Rev. Lett. 77, 5389 (1996).

[20] R.E. Goldstein, T.R. Powers, and C.H. Wiggins, Phys. Rev. Lett. 80, 5232 (1998).
[21] H. Jian, T. Schlick, and A. Vologodskii, J. Mol. Biol. 284, 287 (1998).

[22] G. Chirico and J. Langowski, Biopolymers 34, 415 (1994).

[23] B.A. DiDonna and T.A. Witten, e-print cond-mat/0104119.

[24] P.L. Hansen, D. Svensek, V.A. Parsegian, and R. Podgornik, Phys. Rev. E 60, 1956 (1999). 